\title{
Reduction Mammaplasty as a Treatment Option for Early Breast Cancer
}

\author{
Mohamed A. Shoeib ${ }^{1}$, P. C. Parodi ${ }^{2}$, C. Cidollini ${ }^{3}$ \\ ${ }^{1}$ Plastic Surgery, Sohag University, Sohag, Egypt; ${ }^{2}$ Plastic Surgery, Udine University, Udine, Italy; ${ }^{3}$ General Surgery, Udine Univer- \\ sity, Udine, Italy. \\ Email:mrahmoni@yahoo.com
}

Received July $29^{\text {th }}, 2012$; revised September $1^{\text {st }}, 2012$; accepted October $3^{\text {rd }}, 2012$

\begin{abstract}
Background: Although reduction mammaplasty is a well-known technique for cosmetic purposes, there are few previous reports regarding its application and clinical outcome following conservative breast surgery reconstruction. Mammaplasty combined with breast conservation surgery is an effective treatment of early breast cancer, and leads to better overall results and superior quality of life in patients with previous macromastia or ptosis. Our aim is to analyze both the aesthetic and oncologic outcome of reduction mammaplasty combined with conservative breast surgery reconstruction. Patient and Methods: 25 patients with stage 1 or 2 breast cancer, with a tumor size not more than $3 \mathrm{~cm}$ in its greatest dimension, were operated upon by quadrantectomy, and bilateral reduction mammaplasty. Oncologic data on tumor size, location, and axillary lymph node, were assessed, and accordingly the design of the pedicle was chosen. Reconstructed breast and opposite breast reduction were evaluated. Results: Of the total 50 breast reductions techniques, 35 (70 percent) were superomedial pedicle, 4 ( 8 percent) were superior, 3 ( 6 percent) were superolateral, and 8 (16 percent) were inferior. The cosmetic result was considered to be good or very good in 18 cases, satisfactory in 6 cases, and poor in 1 case. There were no other complications as regard nipple areola ischemia, heamatoma, seroma, wound problems, fat necrosis, or recurrence in any of the cases. Conclusion: By adding breast reduction and other plastic surgery techniques to breast conservation, patients have the opportunity for an improved cosmetic outcome and overall improved quality of life. Although the combined approach between the oncologic surgeon and the plastic surgeon requires more preoperative planning and intraoperative care, the concept can reduce deformities, favor the oncologic treatment, and optimize the aesthetic outcome in most early-stage cancer patients.
\end{abstract}

Keywords: Breast; Reduction; Cancer; Bilateral; Mammaplasty; Conservative

\section{Introduction}

Breast conservation surgery along with radiation has become an accepted alternative to total mastectomy for selected patients with early-stage breast cancer because of its comparable overall survival rate and positive impact on quality of life. The majority of patients treated with breast conservation therapy (BCT) achieve an acceptable cosmetic result with minimal distortion or asymmetry. However, some patients may be left with a significant breast deformity following BCT, thus requiring that they live with disfigurement or undergo reconstruction. Plastic surgeons are available early in the planning stage of this treatment to help anticipate cosmetic problems and attempt to prevent them. The ultimate recommendations regarding surgical, oncological, and reconstructive management depend on the experience and expertise of the surgical team, the location and nature of the tumor, the size and anatomy of the breast, and the overall health and body habitus of the patient [1].

Reduction mammaplasty combined with breast conservation surgery is an effective treatment of early breast cancer [2].

The reduction mammaplasty remains a useful procedure according to the initial breast volume, ptosis, and the amount of glandular tissue resected.

Although outstanding aesthetic outcome may be obtained with all techniques, reduction mammaplasty leads to better overall results and superior quality of life in patients with previous macromastia or breast ptosis [3].

For the tumors located in the lower region of the breast, the tumor resection can be incorporated into the sector of breast tissue removed as part of a superior pedicle mammaplasty. For the tumors located in the inner and outer portions, the reduction pattern can be rotated, and a superior-lateral or a superior-medial pedicle mammaplasty can be prepared [1].

A careful and coordinated planning with the oncologic 
surgeon is important since reduction mammaplasty alters the normal architecture of the breast.

Breast resection with nipple transplantation (free nipple graft procedures) can be used in cases of pronounced breast hypertrophy or if the tumor is located beneath the nipple-areola area.

It is far easier to perform this shape-facilitating and symmetry-enhancing surgical treatment approach at the time of the primary breast conservation procedure than it is to reconstruct the partial mastectomy defect in an irradiated breast.

\section{Patient and Methods}

25 patients with stage 1 or 2 breast cancer, with a tumor size not more than $3 \mathrm{~cm}$ in its greatest dimension, were operated upon by quadrantectomy, and bilateral reduction mammaplasty.

The patients were discovered via regional program of screening. Sentenil lymph node biobsy were done for all of them. The reduction mammaplasty was indicated in patients with medium- or large-volume breasts with or without ptosis.

Oncologic data on tumor size, location, and axillary lymph node, were assessed, and accordingly the design of the pedicle was chosen.

Surgical margins were assessed during surgery by histological examination of frozen sections of the breast specimens while the patient in the operating room.

Axillary lymph node dissections were done according to the positive intra operative Sentenil lymph node biopsy.

Reconstructed breast and opposite breast reduction were evaluated 6 to 18 months.

\section{Surgical Technique}

With the patient sitting, three skin markings are designed: the submammary sulcus, the central meridian of the breast, and the superior point of the new nipple-areola complex. The tumor site is marked and design of its excision is managed to be within the reduction marking to be excised. Local vasoconstriction is performed by infiltrating $0.25 \%$ lidocaine with epinephrine 1:200,000 along the incisions. Excision of the tumor with $10 \mathrm{~mm}$ safety margin and examined by frozen section, and any extra tissue is excised to be sure of the safety margin, also the Sentenil lymph node is harvested and examined by frozen section. If the node is positive, axillary dissection is performed through the mammaplasty incision in medium-volume breasts or through a separate axillary incision in large breasts. The reconstruction initiates with skin undermining beside the tumor bed, permitting wide glandular dissection and enabling advancement of cutaneous glandular flaps into the tumor defect.

The nipple-areola complex is deepithelialized, main- taining the new size of the nipple-areola complex. The demarcated area outside the nipple-areola complex pedicle and within the design is excised and rotated toward its new position. Breast remodeling is usually performed by superior pedicle mammaplasty with a final inverted-T scar.

In instances of tumors located superiorly to the nipple-areola complex, the inferior pedicle mammaplasty is planned. Two drains are inserted (axillary region and breast) and the incisions are closed with interrupted dermal and continuous subcuticular sutures. All patients receive intravenous antibiotics, and oral antibiotics are continued while the drains are in place.

\section{Results}

25 patients were treated with reduction mammaplasty after conservative breast surgery. Of the total 50 breast reductions techniques, 35 (70 percent) were superomedial pedicle, 4 ( 8 percent) were superior, 3 (6 percent) were superolateral, and 8 (16 percent) were inferior. The tumor site was upper medial quadrant in 3 cases, upper lateral in 8 cases, lower medial in 2 cases, and lower lateral in 12 cases (Table 1).

All were observed after adjuvant radiotherapy. Four cases presented prolonged skin lesions (pigmentation disorders, erythema, and telangiectasias) which resolved spontaneously after 6 months.

According to a subjective questionnaire the overall cosmetic result (reconstructed breast and opposite breast) was evaluated.

The cosmetic result was considered to be good or very good in 18 cases, satisfactory in 6 cases, and poor in 1 case (Figures 1-4).

There was no recurrence in a follow up period ranging from 6 to 18 months, but still need further follow up.

There were no other complications as regard nipple areola ischemia, heamatoma, seroma, wound problems, or fat necrosis in any of the cases.

Only one case of infection, and it was cured by oral antibiotics and daily dressing by local antimicrobial ointment for few days.

\section{Discussion}

Reduction mammaplasty combined with breast conserveing surgery is an effective treatment for early breast cancer; however, important technical considerations must be planned in advance. The lack of planning without application of reconstructive techniques can result in aesthetic

Table 1. Site of tumor.

\begin{tabular}{cccc}
\hline $\begin{array}{c}\text { Upper } \\
\text { medial }\end{array}$ & $\begin{array}{c}\text { Upper } \\
\text { lateral }\end{array}$ & $\begin{array}{c}\text { Lower } \\
\text { medial }\end{array}$ & $\begin{array}{c}\text { Lower } \\
\text { lateral }\end{array}$ \\
\hline 3 & 8 & 2 & 12 \\
\hline
\end{tabular}



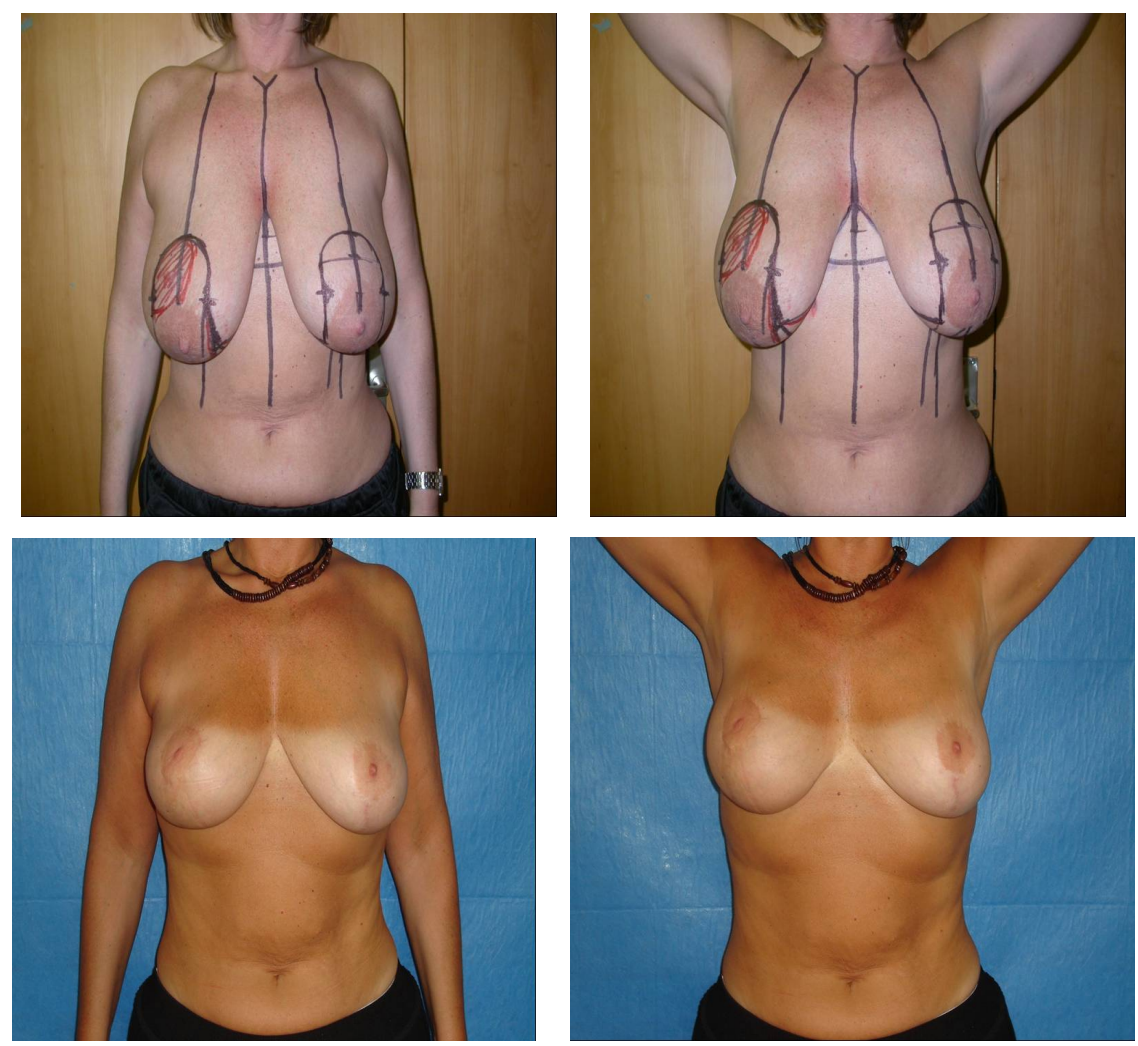

Figure 1. Right upper lateral breast cancer pre and late postoperative.
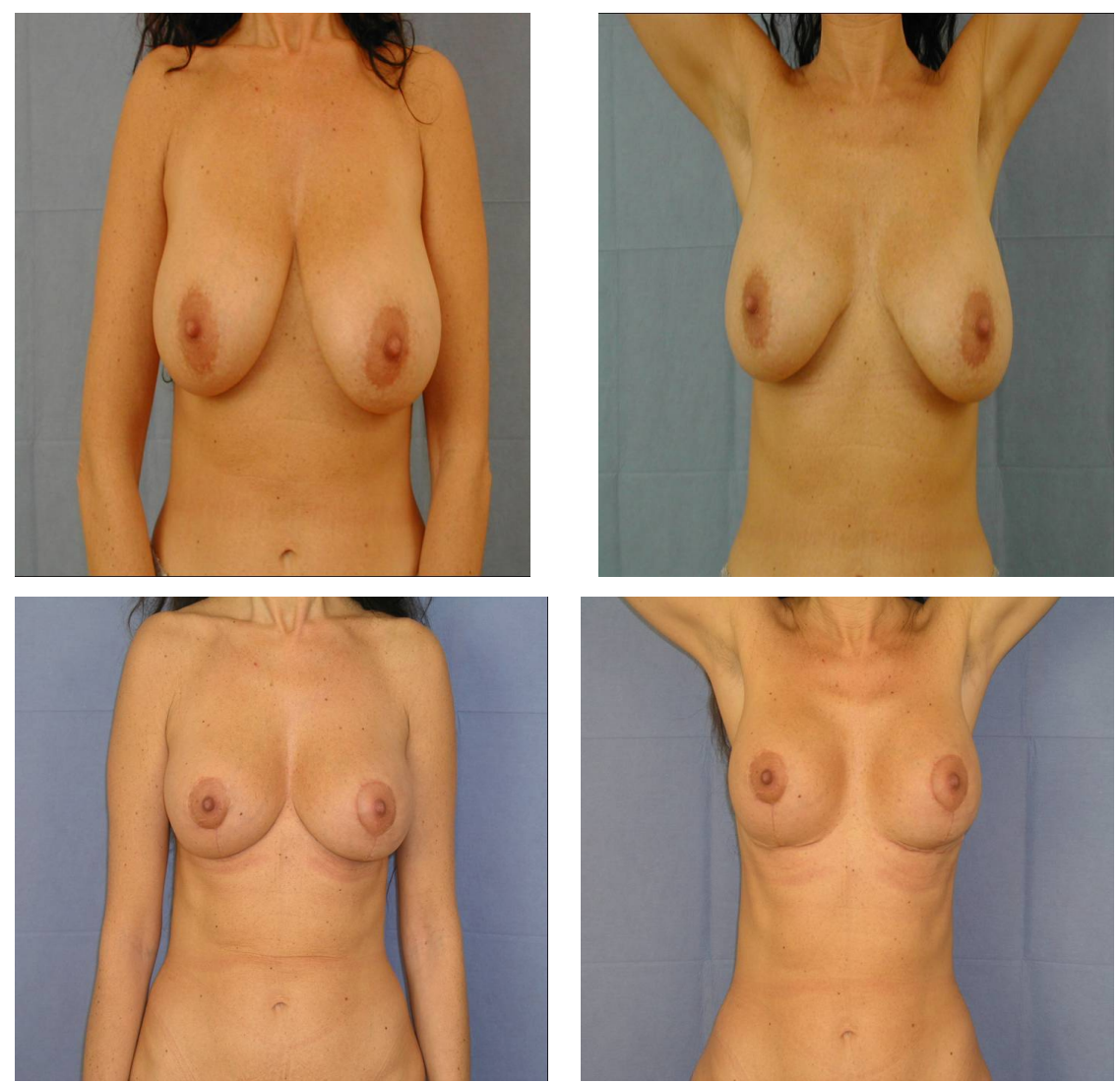

Figure 2. Left lower lateral breast cancer above pre and below late postoperative. 

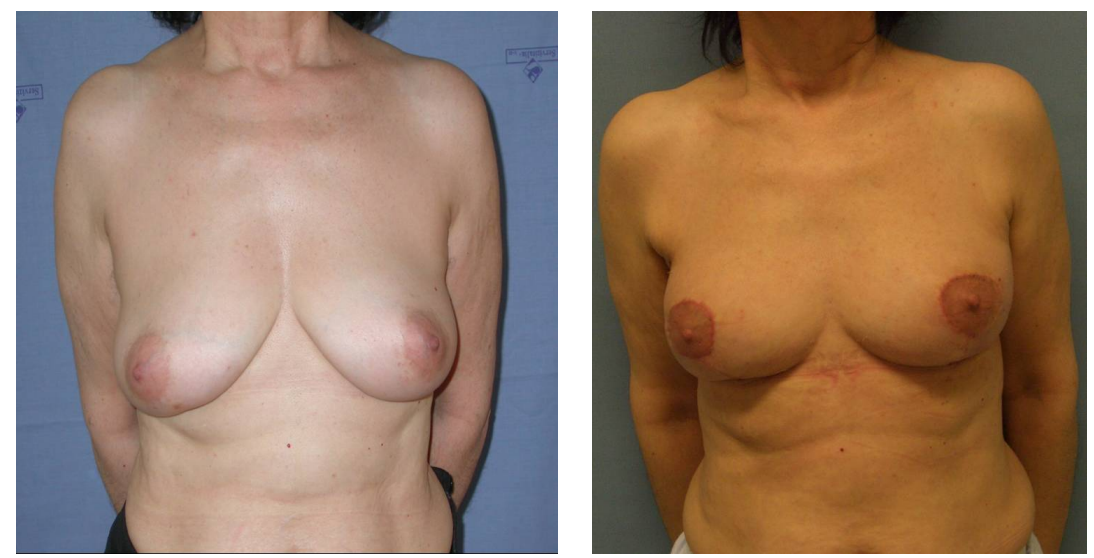

Figure 3. Right lower medial breast cancer left pre and right late postoperative.
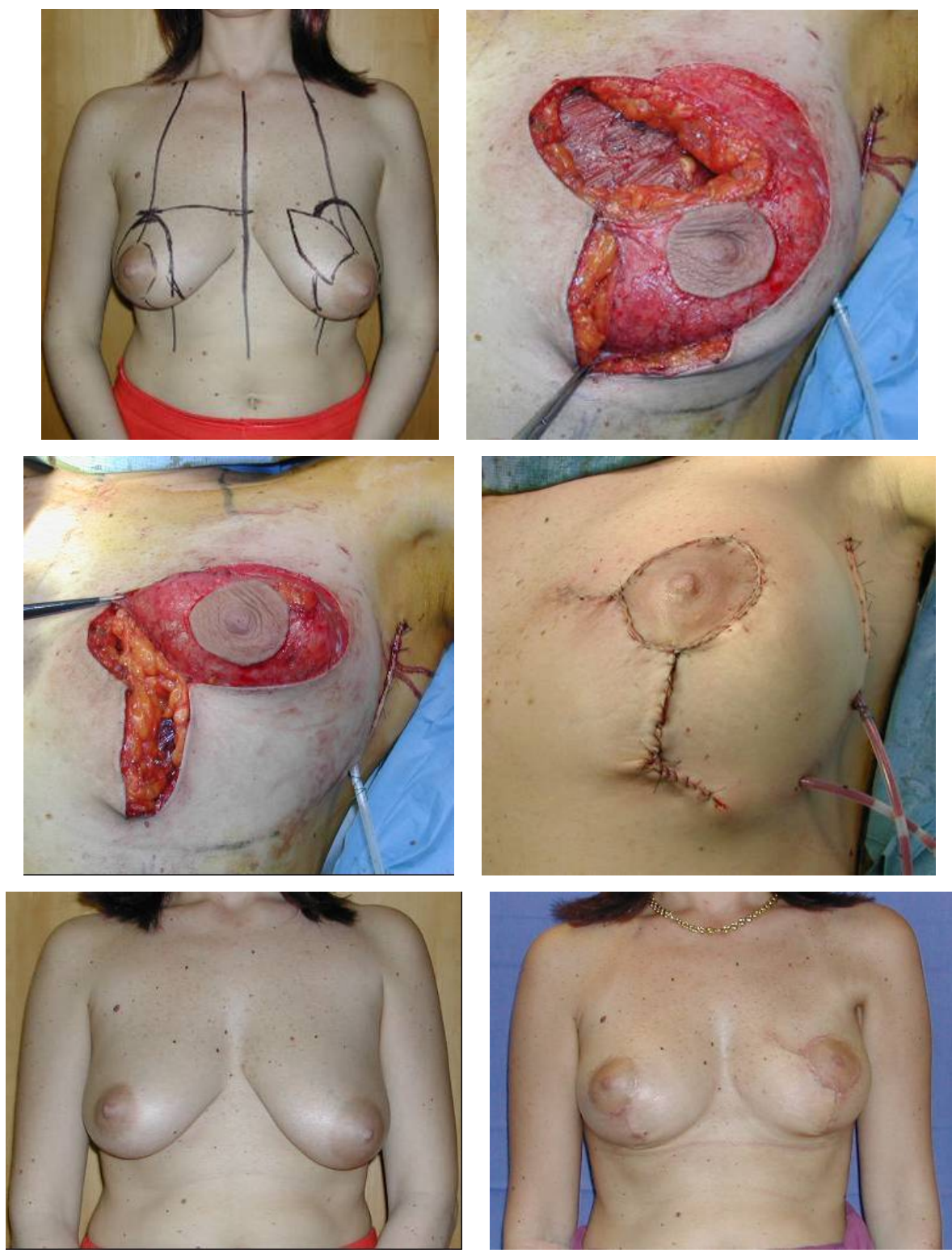

Figure 4. Left upper medial breast cancer above left preoperative marking, above right and middle intraoperative, and below pre and late post operative. 
deformities and patient dissatisfaction.

Preoperative patient evaluation can allow optimal positioning of the biopsy incisions to avoid ischemic flaps.

The integration of plastic surgery techniques at the time of the initial tumor excision for breast reconstructtion is referred to as "oncoplastic surgery", and this is gaining popularity in many specialized breast cancer units, particularly in Europe [4-6].

The option is to propose breast conservative therapy with immediate breast reduction. This can be done by mammaplasty techniques, and the use of different dermoglandular flaps according to the location of the tumor at the time of the initial lumpectomy.

Although the combined approach between the oncologic surgeon and the plastic surgeon requires more preoperative planning and intraoperative care, the concept can reduce deformities, favor the oncologic treatment, and optimize the aesthetic outcome in most early-stage cancer patients.

Several studies have already proven the safety and efficacy of reduction mammaplasty as a breast conservation surgery prior to irradiation of the breast. Newman and colleagues in their study of 28 patients found no local recurrence in their reduction mammaplasty patient population after a median follow-up of 23.8 months [7].

We found no tumor recurrence in any of our patients after 6 to 18 months follow up, beside the good post operative aesthetic outcome, which favor that technique.

Aesthetically pleasing breast reconstruction involves the parameters of symmetry in breast shape and size, reduced scar, and natural appearance, with satisfactory volume restoration [8]. For these reasons we agree with concept of primary surgical interference, the efficacy and safety of performing reduction mammaplasty as an adjunct to breast conservation surgery, and we found that the over all cosmetic outcome after postoperative radiotherapy is not maximumally changed.

\section{Conclusions}

Early diagnosis of breast cancer is a very important prognostic factor and it allows for the introduction of breast conservation therapy, beside the advance in the oncologic treatment.

Screening programs worldwide are responsible for the early detection of breast cancer.
By adding breast reduction and other plastic surgery techniques to breast conservation, patients have the opportunity for an improved cosmetic outcome and overall improved quality of life.

\section{REFERENCES}

[1] A. Losken, E. T. Elwood, T. M. Styblo and J. Bostwick III, "The Role of Reduction Mammaplasty in Reconstucting Partial Mastectomy Defects," Plastic \& Reconstructive Surgery, Vol. 109, No. 3, 2002, pp. 968-975. doi:10.1097/00006534-200203000-00025

[2] A. M. Munhoz, E. Montag, E. G. Arruda, et al., "Critical Analysis of Reduction Mammaplasty Techniques in Combination with Conservative Breast Surgery for Early Breast Cancer Treatment," Plastic \& Reconstructive Surgery, Vol. 117, No. 4, 2006, pp. 1091-1103. doi:10.1097/01.prs.0000202121.84583.0d

[3] E. Chang, N. Johnson, B. Webber, et al., "Bilateral Reduction Mammoplasty in Combination with Lumpectomy for Treatment of Breast Cancer in Patients with Macromastia," American Journal of Surgery, Vol. 187, No. 5, 2004, pp. 647-650. doi:10.1016/j.amjsurg.2004.01.002

[4] N. K. Schondorf, "The Technique of 'B', 'S', or 'W' Reduction Mammaplasty in the Conservative Therapy of Breast Carcinomas: Experience with a New Surgical Technique," Breast, Vol. 10, No. 6, 2001, pp. 501-507. doi:10.1054/brst.1999.0089

[5] A. D. Baildam, "Oncoplastic Surgery of the Breast," British Journal of Surgery, Vol. 89, No. 5, 2002, pp. 532-533. doi:10.1046/j.1365-2168.2002.02077.x

[6] K. B. Clough, J. S. Lewis, B. Couturaud, et al., "Oncoplastic Techniques Allow Extensive Resections for BreastConserving Therapy of Breast Carcinomas," Annals of Surgery, Vol. 237, No. 1, 2003, pp. 26-34. doi:10.1097/00000658-200301000-00005

[7] L. A. Newman, H. M. Kuerer, M. D. McNeese, et al., "Reduction Mammoplasty Improves Breast Conservation Therapy in Patients with Macromastia," American Journal of Surgery, Vol. 181, No. 3, 2001, pp. 215-220. doi:10.1016/S0002-9610(01)00563-3

[8] A. K. Alderman, E. G. Wilkins and J. C. Lowery, "Determinants of Patient Satisfaction in Postmastectomy Breast Reconstruction," Plastic \& Reconstructive Surgery, Vol. 106, No. 4, 2000, pp. 769-776. doi:10.1097/00006534-200009040-00003 